\title{
Correlation between Serum Albumin Level and Severity of Acute Ischemic Stroke - A Prospective Observational Study from Trivandrum, Kerala
}

\author{
Abdul Sameer Edakkara ${ }^{1}$, Ratheesh Narayanan Santhanavally², Ravikumar Kurup ${ }^{3}$ \\ 1,2,3 Department of General Medicine, Government Medical College, Thiruvananthapuram, Kerala, India.
}

\section{ABSTRACT}

\section{BACKGROUND}

Preclinical studies have shown a neuroprotective effect of albumin in acute ischemic stroke (AIS). Owing to the scarcity of clinical studies establishing the correlation of serum albumin levels and AIS, the present study was conducted using National institute of health stroke scale score (NIHSS).

\section{METHODS}

The present prospective observational study evaluated the association of serum albumin and NIHSS score at baseline, 1 week, 1 month and 3 months of admission in 18 participants aged 18 years or more admitted within 48 hours of AIS in Internal Medicine Department of Government Medical College, Trivandrum between 2019 and 2020. Participants with transient ischemic attack, atrial fibrillation, decompensated cirrhosis of liver, nephrotic syndrome, hypoalbuminemia, hypercholesterolemia, and intracranial hematoma were excluded. Institutional Ethics Committee approved the project and written informed consent was obtained from all study participants. Serum albumin was estimated using enzyme linked immunosorbent assay (ELISA) kit using standardized technique. Logistic regression was used to determine the association of albumin and NIHSS. Analysis was done using $\mathrm{R}$ and $\mathrm{p}<0.05$ was considered statistically significant.

\section{RESULTS}

The mean age of study participants was 60.2 (12.9) and $55.6 \%$ participants were males. $11.1 \%, 5.6 \%$ and $22.2 \%$ participants had history of ischemic stroke, chronic kidney disease, and coronary artery disease respectively. At baseline serum albumin was 3.5 (1) g/dL and NIHSS was 13.2 (9.3). Negative correlation between serum albumin and NIHSS scale at baseline $(r=-0.873, p<0.01)$, at one week $(r=-$ 0.856 and $\mathrm{p}<0.01)$, at one month $(\mathrm{r}=-0.856$ and $\mathrm{p}<0.01)$ and three months $(\mathrm{r}=-$ 0.856 and $\mathrm{p}<0.01$ ) was observed.

\section{CONCLUSIONS}

Lower serum albumin level was associated with severe neurological deficit and had worse outcomes in patients with acute ischemic stroke. Albumin can be used as an easy, inexpensive, rapid, and reliable tool in determining the prognosis of AIS.

\section{KEY WORDS}

Serum Albumin, Acute Ischemic Stroke, Outcome, Prognosis, NIHSS
Corresponding Author:

Dr. Ratheesh Narayanan Santhanavally, Associate Professor,

Department of General Medicine,

Government Medical College,

Thiruvananthapuram, Kerala, India.

E-mail: ratishkarthika@yahoo.co.in

DOI: $10.14260 / \mathrm{jemds} / 2021 / 780$

How to Cite This Article:

Edakkara AS, Santhanavally RN, Kurup R. Correlation between serum albumin level and severity of acute ischemic stroke - a prospective observational study from Trivandrum, Kerala. J Evolution Med Dent Sci 2021;10(45):3859-3863, DOI: $10.14260 /$ jemds $/ 2021 / 780$

Submission 02-11-2021,

Peer Review 09-11-2021,

Acceptance 02-12-2021,

Published 21-12-2021.

Copyright (c) 2021 Abdul Sameer Edakkara et al. This is an open access article distributed under Creative Commons Attribution License [Attribution 4.0 International (CC BY 4.0)] 


\section{BACKGROUND}

Stroke is a major cause of mortality and morbidity. Stroke is the second most common cause of death globally and accounts for 5.7 million deaths each year. ${ }^{1}$ More than $85 \%$ of the global burden of stroke and its related complications are from the low and middle-income countries ${ }^{2}$ according to 2001 Global burden of disease survey. The dramatic rise in the population of elderly due to the advancements in medical and surgical fields and also the improvement in quality of life along with increase in prevalence of lifestyle diseases such as diabetes mellitus, hypertension, dyslipidaemia and obesity could be attributed to the increase in incidence of stroke and the related complications. A systematic review observed a prevalence rate of stroke of 45 - 487 in urban areas and 55 388.4 in rural areas per lakh in India. ${ }^{3}$ The high prevalence of lifestyle diseases such as diabetes mellitus, metabolic syndrome, obesity and systemic hypertension in urban population and also the increasing prevalence even in rural population has been attributed to the increase in this prevalence of a decade. This could also mean as the increase in recognition of these diseases in rural areas of the country as well. Timely intervention, appropriate measures to control the risk factors, early mobilization, treatment, and physiotherapy are cornerstones of treatment of stroke. Since the burden of stroke and stroke related mortality and morbidity is increasing in India, proper encouragement in research works that deal with diagnosis, management and follow up of stroke is the need of the hour.

A stroke is a hasty neurological blow-up due to impaired perfusion of brain. Blood vessels with clots interrupt the blood flow to the brain and ultimately leads to the rupture of the vessels. ${ }^{4}$ Stroke is a rapidly developing clinical signs of focal (or global) disturbance of cerebral function, with symptoms lasting 24 hours or longer or leading to death, with no apparent cause other than that of vascular origin. ${ }^{5}$ Stroke is broadly classified into ischemic and haemorrhagic types. It is also classified according to the anatomical area involved. Ischemic stroke is due to deficient blood supply to the brain and haemorrhagic stroke is due to intracranial haemorrhage. Ischemic stroke can be classified into large vessel stroke, cardio embolic stroke, and small vessel stroke. ${ }^{5}$ Acute ischemic stroke is the occlusion of cerebral blood vessels by either a thrombus or emboli subsequently leading to neuronal damage and cell death. Stroke is also a leading cause of the disability, a large number of stroke survivors have neurological deficits resulting in disabilities that affect the quality of their life. ${ }^{6}$ Age, race, gender, ethnicity and genetic predisposition are the non-modifiable risk factors while diabetes mellitus, hypertension, cardiac disease, high cholesterol and sedentary lifestyle are the modifiable risk factors for stroke. ${ }^{7}$

Early diagnosis and management of stroke reduce the morbidity and mortality associated with it. Currently, brain imaging is considered as the gold standard diagnostic procedure and also to assess the improvement in follow up management of stroke. ${ }^{8}$ Higher cost and lower availability in remote rural areas are major barriers to the gold standard investigation especially in developing and low-income countries including India. The evaluation of various biochemical markers and their utility in predicting the mortality and morbidity and determining the progression of disease has proven futile. ${ }^{9}$ Ongoing studies are focusing on determining a cost-effective, highly reliable and easily accessible biomarker for stroke. Human albumin constitutes $50 \%$ of the total body protein and is a single peptide chain consisting of 585 amino acids. ${ }^{10}$ Body fluids contain approximately 300 - $500 \mathrm{~g}$ of albumin. Albumin maintains the plasma oncotic pressure and is a major carrier in the blood. Human albumin is currently used for the management of burns, hypovolemic stroke, hepatorenal syndrome, bacterial peritonitis etc. Previous literature has demonstrated that occurrence of stroke is indirectly related to the serum albumin levels. ${ }^{11}$ Animal studies have showed the beneficial effect of albumin therapy in transient focal ischaemia ${ }^{12}$ and has also shown neuroprotective effects in focal cerebral ischemia. ${ }^{13}$ This is probably due to the inhibitory effects of albumin against leukocyte adhesion within the post capillary microvasculature and thrombosis in the early reperfusion phase. The promotion of collateral formation in ischemic areas is influenced by the serum albumin levels. ${ }^{14}$ The prognostic significance of serum albumin in acute ischemic stroke ${ }^{15}$ and local administration of low dose cold albumin infusion therapy has shown neuroprotective effects.

The National institute of health stroke scale is a tool used by health professionals to quantify the impairments occurring in stroke. ${ }^{16}$ NIHSS consist of 11 parameters which are given individual score that ranges between 0 to 4 , where 0 indicates normal function and 4 is severe impairment. The maximum score is 42 while the lowest possible score is zero. NIHSS is a reliable tool for assessing stroke severity. ${ }^{17}$ Serum albumin levels and its correlation to stroke and its outcomes have been a hot scientific discussion topic for decades. Till date there has not been any conclusive evidence establishing the role of serum albumin and stroke severity, stroke related complications, mortality, and morbidity. These lacunae of research have encouraged us to do the present study with aims of establishing concrete evidence determining the role of serum albumin level in AIS.

\section{METHODS}

This prospective observational study was conducted at the Government Medical College and Hospital Thiruvananthapuram, Kerala from 2019 to 2020 after getting approval from the Institutional Ethics Committee (IEC number: $15 / 28 / 2018 / \mathrm{MCT})$. The study was conducted as per the Declaration of Helsinki, GCP guidelines and ICMR guidelines for Biomedical and Health research involving human participants. Sample size was calculated and fixed as 18 assuming an $\alpha$ error of $0.05, \beta$ error of 0.2 and $80 \%$ power. ${ }^{18}$ All participants aged 18 years or above admitted within 48 hours of onset of symptoms of stroke were included. Participants with the transient ischemic attack, cardiac diseases with atrial fibrillation, decompensated cirrhosis of the liver, nephrotic syndrome (proteinuria of more than $3.5 \mathrm{gm} /$ day), hypoalbuminemia $(<3.5 \mathrm{~g} / \mathrm{dL}$ ), hyper-cholesterolaemia (total cholesterol of more than 200 $\mathrm{mg} / \mathrm{dl}$ and pitting oedema), haematoma on CT brain and protein losing enteropathy were excluded from our study. Data was collected in interviewer administered semistructured questionnaire. A non-contrast CT brain was taken as a part of routine diagnostic investigation ${ }^{19}$ along with 
serum albumin. Routine neurological examination, NIHSS at the time of admission, at one week, one month, 2 months and 3 months of onset of symptoms were recorded in the case record form. Serum albumin less than $3.5 \mathrm{~g} / \mathrm{dl}^{20}$ was considered as hypoalbuminemia and was determined within 24 hours of admission at the central laboratory using the ELISA kit using standardized technique. Participants were enrolled after obtaining written informed consent.

\section{Statistical Analysis}

Data was analyzed using $\mathrm{R}$, regression analysis was used to determine the association between serum albumin and NIHSS at baseline and follow up points. Between groups, comparison was made using independent sample $t$ test. Normality of data was assessed using Shapiro-Wilk test and tests of significance were independent sample t test and chi square test. Data is expressed as mean [standard deviation (SD)] after rounding off to single decimal. P $<0.05$ was considered statistically significant.

\section{RESULTS}

The mean age of the study participants was 60.2 (12.9) years. $55.6 \%(\mathrm{n}=10)$ participants were males and $44.4 \%(\mathrm{n}=8)$ were females. The baseline characteristic of the study participants is described in table 1. Participants with history of ischemic stroke, chronic kidney disease, and coronary artery disease were $11.1 \%, 5.6 \%$ and $22.2 \%$ respectively. Baseline serum albumin was 3.5 (1) g/dL and NIHSS was 13.2 (9.3). Baseline serum albumin $(\mathrm{p}=0.009)$ and NIHSS $(\mathrm{p}=$ 0.048 ) was significantly lower in females.

\begin{tabular}{|cccc|}
\hline Parameter & $\begin{array}{c}\text { Male Mean } \\
\text { (SD) }\end{array}$ & $\begin{array}{c}\text { Female Mean } \\
\text { (SD) }\end{array}$ & P \\
\hline Age (years) & $61.4(5.8)$ & $59.4(6.9)$ & 0.06 \\
Systolic blood pressure (mmHg) & $158.5(22.1)$ & $149.9(26.7)$ & 0.08 \\
Diastolic blood pressure (mmHg) & $84.6(16.4)$ & $86.7(13.3)$ & 0.2 \\
Serum albumin (g/dl) & $3.7(0.6)$ & $3.3(0.4)$ & $0.009^{*}$ \\
NIHSS & $14.1(9.9)$ & $12.7(8.6)$ & $0.048^{*}$ \\
Haematocrit (\%) & $42.2(5.8)$ & $41.4(6)$ & 0.4 \\
\hline Table 1. Baseline Characteristics of the Study Participants \\
\hline *indicates significant difference between the groups using independent sample t test \\
\hline
\end{tabular}

Participants with higher serum albumin at baseline had significantly lower NIHSS at baseline. The NIHSS at different time periods is demonstrated in figure 1.

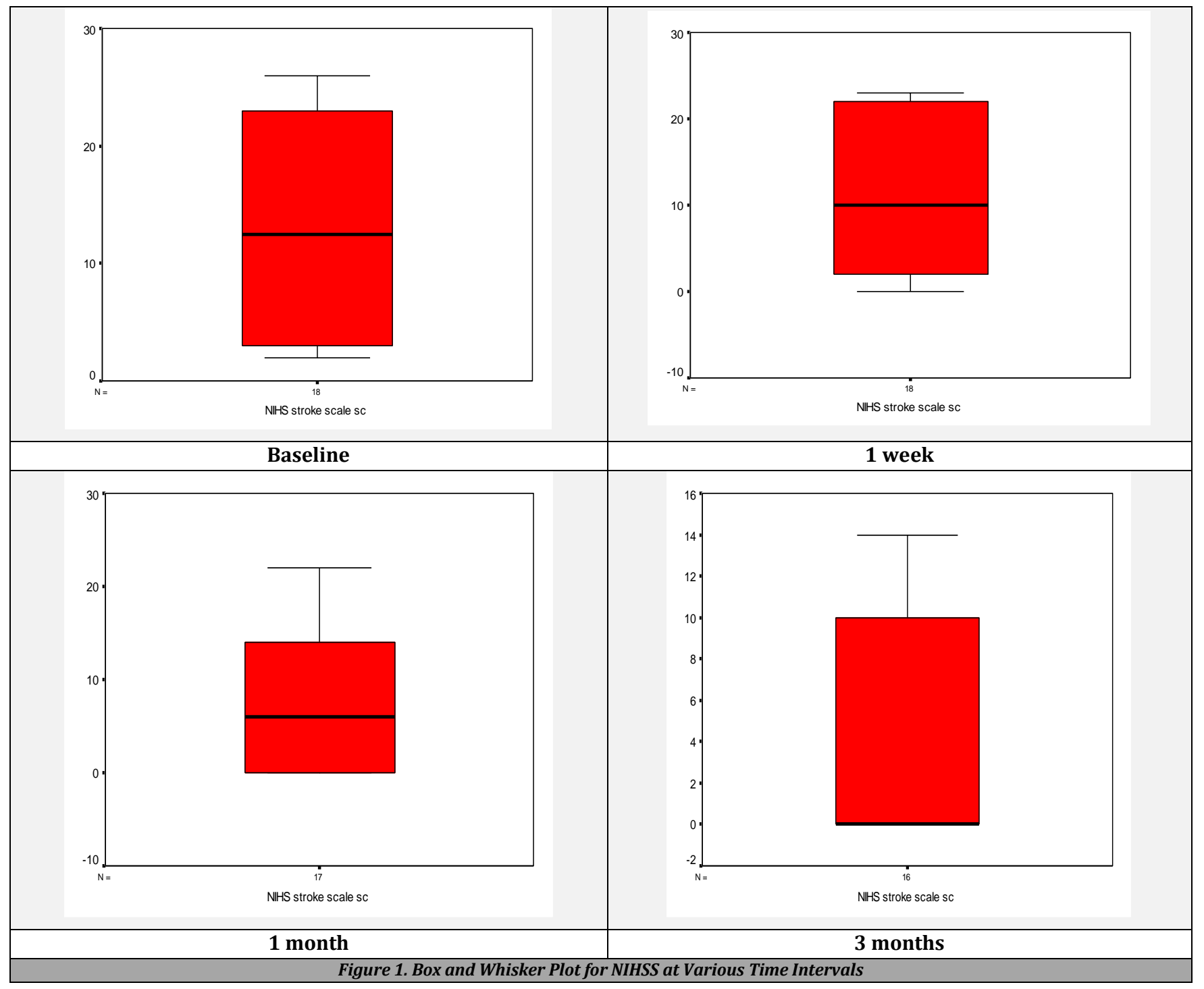


Significant negative correlation between serum albumin and NIHSS at baseline was observed, Karl Pearson correlation coefficient $(\mathrm{r})=-0.873(\mathrm{p}<0.01)$. Correlation between serum albumin and NIHSS is demonstrated in table 2 .

\begin{tabular}{|ccc|}
\hline Time & $\mathbf{R}$ & $\mathbf{P}$ \\
1 week & $-0.856^{*}$ & $<0.01$ \\
1 month & $-0.876^{*}$ & $<0.01$ \\
3 months & $-0.856^{*}$ & $<0.01$ \\
\hline Table 2. Correlation between Basal serum Albumin Level and the \\
Severity of Acute Ischemic Stroke as Assessed by NIHSS Score at \\
Different time Intervals
\end{tabular}

\section{DISCUSSION}

The present prospective observational study investigated the association between serum albumin at the time of admission and its effect on the outcome of ischemic stroke, measured by NIHSS at admission, 1 week, 1 month and 3 months of onset of symptoms. The scarcity of published literature on the prognostic efficacy of albumin as a surrogate marker to assess the severity of stroke and predicting the outcomes of stroke will open new dimensions in stroke-related medical science. The mean age of the study participants was $\sim 60$ years which is higher than the mean age of the reference study where it was $\mathbf{5 7 . 5}$ years and other published literature was 57.7 years. ${ }^{21}$ This could be attributed to the high standards of healthcare of Kerala and the egalitarian healthcare facilities of the state. ${ }^{18}$ Age is the most important non-modifiable risk factor; with advancing age, incidence of stroke, morbidity and mortality associated with it also progress. The risk of stroke rate doubles both genders after each successive decade after 55 years of age. Approximately $76 \%$ strokes occur in individuals aged 65 years ${ }^{22,23}$ or above. During the hospital stay, serum albumin could reduce due to lack of appropriate nutrition. Hospital-acquired infections could also reduce the level of serum albumin. Hence to estimate the baseline serum albumin levels, it was estimated at the time of admission. ${ }^{18}$ Baseline serum albumin was 3.5 (1) g/dL and the baseline NIHSS was 13.2 (9.3). The NIHSS reduced to 11.8 (9), 7.9 (7.9) and 4.5 (5.5) at 1 week, 1 month and 3 months of onset of symptoms.

In our study those who had higher serum albumin had low NIHS stroke scale score at the time of admission. On follow up, we found out that there is a significant negative correlation between serum albumin level and NIHS stroke scale score after 1 week, after 1 month and after 3 months. Previous literatures have demonstrated that the participants with poor functional outcomes had significant lower serum albumin according to modified Rankin scale. ${ }^{8}$ Similarly, another publication concluded that low levels of albumin was associated with poor outcome and a higher chance of stroke recurrence and death rate compared to participants with normal or elevated levels of serum albumin. Lower levels of serum albumin was significantly associated with poor outcomes in all the stroke subtypes classified according to TOAST (Trial of ORG 10172 in Acute Stroke Treatment). ${ }^{24}$ In contradiction to these findings, a phase 3, randomized, double blinded placebo-controlled pilot trial failed to demonstrate the beneficial effect of albumin infusion in ischemic stroke and a significant adverse event, pulmonary oedema ${ }^{25}$ reduced the validity of the use of albumin.

From our study it is evident that serum albumin plays a major role in the clinical outcome of acute ischemic stroke. By increasing low shear viscosity and decreasing erythrocyte sedimentation under no-flow conditions serum albumin reduces the haematocrit level and also plays a preventive role in aggregation of erythrocytes. Albumin also has antioxidant property. Albumin captures the oxygen free radicals and slows the production of reactive hydroxyl radical species. Other proposed mechanisms include prevention of thrombosis and prevention of leukocyte adhesion within the capillaries. ${ }^{8}$ In support of this, a prospective controlled study demonstrated that lower albumin levels in patients with cardio embolic stroke was associated with a high rate of mortality. ${ }^{26}$ Free lysophosphatidylcholine increases leukocyte adhesion molecules which leads to inflammatory damage of vascular endothelium. When present in high concentrations, lysophosphatidylcholine also produces apoptosis. Interestingly, it is known that albumin can bind to lysophosphatidylcholine and inhibit them subsequently. Depending on these findings albumin infusion after ischaemic stroke may be beneficial for a better long-term outcome but further studies are required in this province.

\section{CONCLUSIONS}

Significant negative correlation was observed between basal serum albumin level and the severity of acute ischemic stroke at baseline, 1 week, 1 month and 3 months of admission. Low baseline serum albumin had severe neurological deficit and the worst prognosis over time in patients with acute ischemic stroke. These findings suggest that serum albumin has to be estimated in all patients with AIS to understand the severity, predict the complications, mortality, morbidity and recovery period.

\section{Limitations of the Study}

Small sample size and use of only NIHSS are the major limitations of the present study and the radiological correlation such as infarct size and the resolution of symptoms and recovery on follow up was not assessed in the present study. Further long-term prospective studies addressing these limitations are required to establish the conclusive role of serum albumin level in ischemic stroke.

Data sharing statement provided by the authors is available with the full text of this article at jemds.com.

Financial or other competing interests: None.

Disclosure forms provided by the authors are available with the full text of this article at jemds.com.

\section{REFERENCES}

[1] Stroke Talk 1.pdf [Internet]. http://www.strokeindia.org/downloads/Stroke \%20Talk \%201.pdf

[2] Strong K, Mathers C, Bonita R. Preventing stroke: saving lives around the world. Lancet Neurol 2007;6(2):182-7. 
[3] Khurana S, Gourie-Devi M, Sharma S, et al. Burden of stroke in India during 1960 to 2018: a systematic review and meta-analysis of community based surveys. Neurol India 2021;69(3):547-59.

[4] Kuriakose D, Xiao Z. Pathophysiology and treatment of stroke: present status and future perspectives. Int J Mol Sci 2020;21(20):7609.

[5] Baseline NIH stroke scale score strongly predicts outcome after stroke: a report of the Trial of Org 10172 in acute stroke treatment (TOAST).Neurology 1999;53(1):126-31.

[6] Gresham GE, Fitzpatrick TE, Wolf PA, et al. Residual disability in survivors of stroke--the Framingham study. N Engl J Med 1975;293(19):954-6.

[7] Chugh C. Acute ischemic stroke: management approach. Indian J Crit Care Med 2019;23(Suppl 2):S140-6.

[8] Gad MS, Zakaria NHED, Elgayar NHAAM. Evaluation of the role of ischemia modified albumin as a new biochemical marker for differentiation between ischemic and hemorrhagic stroke. Alexandria Journal of Medicine 2015;51(3):213-7.

[9] Kumar KA, Uthappa S, Surendran S, et al. Correlation of albumin concentration and ischemia modified albumin in the diagnosis of acute myocardial infarction. International Journal of Biomedical and Advance Research 2015;6(4):310-5.

[10] Caraceni P, Tufoni M, Bonavita ME. Clinical use of albumin. Blood Transfus 2013;11(Suppl 4):s18-25.

[11] Boselli M, Aquilani R, Baiardi P, et al. Supplementation of essential amino acids may reduce the occurrence of infections in rehabilitation patients with brain injury. Nutr Clin Pract 2012;27(1):99-113.

[12] Belayev L, Busto R, Zhao W, et al. Effect of delayed albumin hemodilution on infarction volume and brain oedema after transient middle cerebral artery occlusion in rats. J Neurosurg 1997;87(4):595-601.

[13] Belayev L, Zhao W, Pattany PM, et al. Diffusion-weighted magnetic resonance imaging confirms marked neuroprotective efficacy of albumin therapy in focal cerebral ischemia. Stroke 1998;29(12):2587-99.

[14] DeFazio RA, Zhao W, Deng X, et al. Albumin therapy enhances collateral perfusion after laser-induced middle cerebral artery branch occlusion: a laser speckle contrast flow study. J Cereb Blood Flow Metab 2012;32(11):2012-22.
[15] Makris K, Koniari K, Spanou L, et al. Prognostic significance of serum albumin level changes in acute ischemic stroke: the role of biological and analytical variation. Clin Chem Lab Med 2016;54(1):143-50.

[16] Lyden P. Using the National Institutes of Health Stroke Scale: a cautionary tale. Stroke 2017;48(2):513-9.

[17] NIH_Stroke_Scale_508C.pdf [cited 2021 Sep 29]. https://www.stroke.nih.gov/documents/NIH_Stroke_Sc ale_508C.pdf

[18] Sandeep F, Bai P, Kumari R, et al. Prognostic significance of serum albumin in acute ischemic stroke. EJPMR 2017;4(8):138-42.

[19] Muir KW, Weir CJ, Murray GD, et al. Comparison of neurological scales and scoring systems for acute stroke prognosis. Stroke 1996;27(10):1817-20.

[20] Busher JT. Serum Albumin and Globulin. In: Walker HK, Hall WD, Hurst JW, eds. Clinical methods: the history, physical, and laboratory examinations. $3^{\text {rd }}$ edn. Boston: Butterworths 1990.

[21] Abubakar S, Sabir A, Ndakotsu M, et al. Low admission serum albumin as prognostic determinant of 30-day case fatality and adverse functional outcome following acute ischemic stroke. Pan Afr Med J 2013;14:53.

[22] Feigin VL, Lawes CMM, Bennett DA, et al. Stroke epidemiology: a review of population-based studies of incidence, prevalence, and case-fatality in the late 20th century. Lancet Neurol 2003;2(1):43-53.

[23] Rosamond W, Flegal K, Furie K, et al. Heart disease and stroke statistics--2008 update: a report from the American Heart Association Statistics Committee and Stroke Statistics Subcommittee. Circulation 2008;117(4):e25-146.

[24] Babu MS, Kaul S, Dadheech S, et al. Serum albumin levels in ischemic stroke and its subtypes: correlation with clinical outcome. Nutrition 2013;29(6):872-5.

[25] Ginsberg MD, Palesch YY, Hill MD, et al. High-dose albumin treatment for acute ischaemic stroke (ALIAS): a randomised, double-blind, phase 3, placebo-controlled trial. Lancet Neurol 2013;12(11):1049-58.

[26] Alvarez-Perez FJ, Castelo-Branco M, Alvarez-Sabin J. Albumin level and stroke. Potential association between lower albumin level and cardioembolic aetiology. Int J Neurosci 2011;121(1):25-32. 